\title{
Proprioceptive Inputs to Nonspiking Local Interneurons Contribute to Local Reflexes of a Locust Hindleg
}

\author{
M. Burrows, G. J. Laurent and L. H. Fielda \\ Department of Zoology, University of Cambridge, Cambridge CB2 3EJ, England
}

Local reflexes of a leg of the locust Schistocerca gregaria (Forskal) can be elicited by selective stimulation of a proprioceptor (the femoral chordotonal organ) at the femorotibial joint. Motor neurons are either excited or inhibited, so that a coordinated reflex response of a leg results. At the same time, some nonspiking local interneurons are either excited or inhibited by the inputs from these proprioceptive afferents. Altering the membrane potential of an individual, nonspiking interneuron can either increase or decrease the response of the participating motor neurons to the proprioceptive stimulus and thereby alter the gain of the reflex. To determine the pathways, and to understand the role of the nonspiking interneurons in mediating these reflex effects, recordings were made simultaneously from these iriterneurons and afferent neurons.

The excitation of a particular nonspiking local interneuron is produced monosynaptically by the afferent neurons. Chemically mediated EPSPs consistently follow sensory spikes with a latency that is the same as that for the known parailel, direct connections made by these sensory neurons with motor neurons (Burrows, 1987a). The chordotonal afferents and the branches of the local interneurons project to the same regions of neuropil. In contrast, the simplest inhibitory pathway is disynaptic, involving spiking local interneurons. The afferents make direct excitatory connections with some of these spiking interneurons, which then make direct inhibitory connections with a nonspiking interneuron. Interactions between the local interneurons add to the complexity of the pathways. For example, excitation of a nonspiking interneuron may also result from disinhibition between spiking local interneurons and inhibition from other nonspiking interneurons.

These patterns of connection provide a sufficient explanation for the differing responses of the various nonspiking interneurons to the proprioceptive stimuli. Some interneurons receive a purely excitatory input and show a sensitivity to movement in one or both directions. By contrast, other interneurons receive a purely inhibitory input, but, again, can show a directional sensitivity, with the depolarizing phase

\footnotetext{
Received Sept. 26, 1987; revised Nov. 16, 1987; accepted Nov. 27, 1987.

This work was supported by grants from the SERC (UK) and the Wellcome Foundation. We thank our colleagues for their many helpful suggestions during the course of this work.

Correspondence should be addressed to Professor M. Burrows, Department of Zoology, University of Cambridge, Downing Street, Cambridge CB2 3EJ, England. Permanent address: Department of Zoology, University of Canterbury, Christ. church 1, New Zealand.

Copyright (c) 1988 Society for Neuroscience $0270-6474 / 88 / 083085-09 \$ 02.00 / 0$
}

of their response resulting from disinhibition. Still other interneurons receive both inhibitory and excitatory inputs and are depolarized by one direction of movement and hyperpolarized by the other.

A crucial role for the nonspiking interneurons in controlling local reflexes is indicated by these results. They may also be sites for the convergence of coordinating signals from other segments, or for the alteration of afferent signals during voluntary movement.

Stimulation of particular receptors of a limb can lead to specific reflex movements that adjust the posture or pattern of locomotion of the animal. To understand how these reflexes are controlled requires, first, that the synaptic connections formed by the participating neurons be revealed. This task is simplified if the sensory and motor neurons involved in the reflexes are few and identifiable. In the legs of insects, a chordotonal organ signals position or movement of the tibia about the femur (locust: Usherwood et al., 1968; Zill, 1985a; stick insect: Hofmann et al., 1985). It initiates reflexes in a small group of identified leg motor neurons so that an imposed movement can be resisted and adjustments made to other joints of the same leg (locust: Burrows and Horridge, 1974; Field and Rind, 1981; stick insect: Bässler, 1972a, b).

These reflexes occur when the animal is standing normally (Zill, 1987) and when it is walking (Cruse, 1981; Cruse and Pflüger, 1981). They are reliable in a particular posture but may become more variable and reverse in sign if excitability changes (Bässler, 1976, 1986; Zill, 1985b). In locusts, some of the pathways mediating these reflex effects can be explained. For example, receptors of the chordotonal organ, excited by an imposed extension of the tibia, directly excite flexor tibiae motor neurons so that the movement can be resisted (Burrows, 1987a). At the same time, these afferents also excite particular spiking local interneurons (Burrows, 1987a) that inhibit either the antagonistic extensor tibiae motor neurons (Burrows and Siegler, 1982) or nonspiking interneurons that would excite them (Burrows, 1987b). Thus, agonist motor neurons are excited monosynaptically, while antagonists are inhibited by more complex pathways involving one or 2 layers of local interneurons. These pathways fail to explain all the observed effects in the motor neurons and omit a role for the nonspiking interneurons. These interneurons excite or inhibit the motor neurons by means of the graded release of transmitter (Burrows and Siegler, 1978), and control them in sets appropriate for the performance of coordinated movements (Burrows, 1980). When the joints of a leg are moved, the membrane potential of some of these inter- 
Figure 1. Nonspiking interneurons mediate reflexes initiated by proprioceptive signals from the FCO. $A, \mathrm{~A}$ resistance reflex. Flexion of the FCO excites the slow extensor tibiae and, at the same time, a nonspiking interneuron is depolarized. Extension of the $\mathrm{FCO}$ excites an antagonistic flexor tibiae motor neuron and is accompanied by a repolarization of the interneuron. $B, A$ steady hyperpolarization applied to the interneurnn enhances the amplitude of its depolarization, but reduces the reflex response of the extensor motor neuron to an imposed flexion. $C$, An interjoint reflex. A flexion of the FCO excites the levator tarsi motor neuron (spikes are recorded from N5b2 in the distal femur) so that the tarsus is levated and, at the same time, a nonspiking interneuron is depolarized. $D$, A steady depolarization applied to the interneuron enhances the reflex excitation of the levator motor neuron. Calibration: vertical, $4 \mathrm{mV}$ (interneuron $A$ ); $10 \mathrm{mV}(B-D)$; movement, $50^{\circ}$.
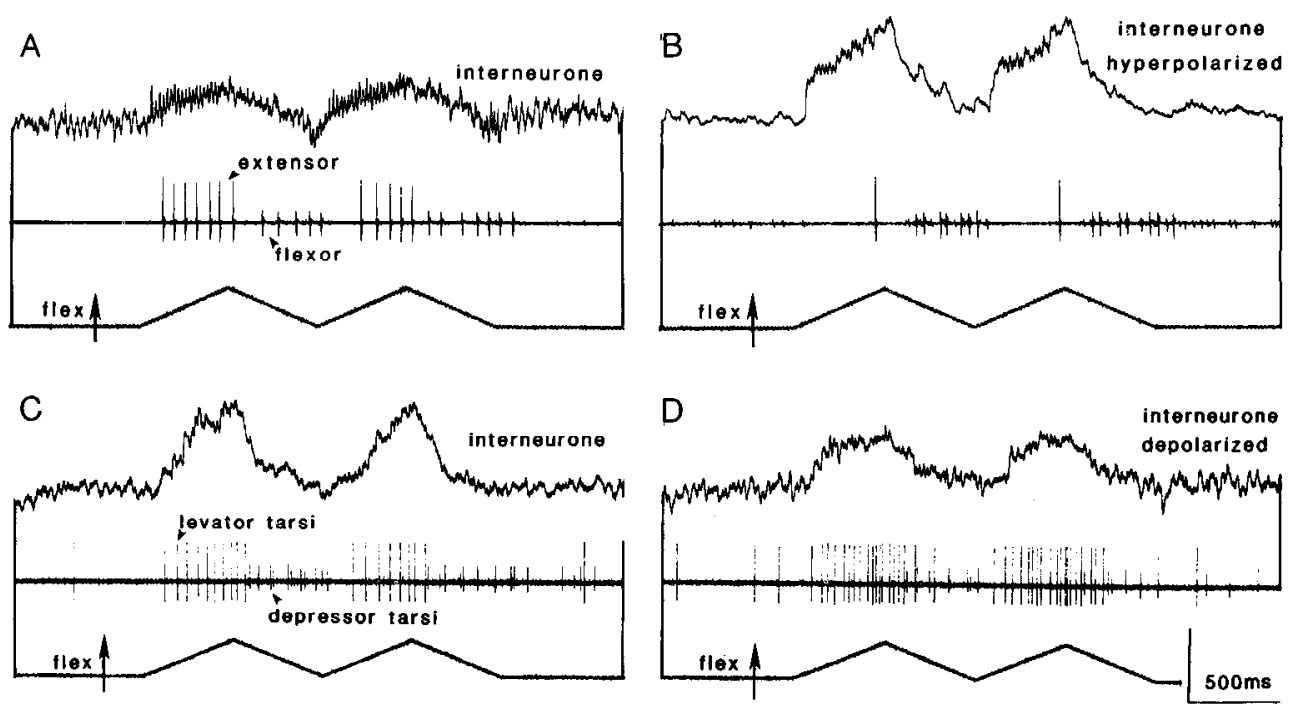

neurons is altered (Siegler, 1981a). In turn, changing the membrane potential of a nonspiking interneuron can alter the strength of a reflex evoked by these movements (Siegler, 1981b). In an abstract, Zill (1981) described some excitatory effects on nonspiking interneurons when the femoral chordotonal organ was moved.

In this paper we show that nonspiking interneurons are essential elements in reflexes initiated by the femoral chordotonal organ. A single interneuron can alter the gain of a reflex when its membrane potential is manipulated experimentally. The excitation of the nonspiking interneurons during these reflexes is mediated by direct afferent connections, while the inhibition results from disynaptic pathways involving spiking local interncurons.

\section{Materials and Methods}

Adult male or female locusts, Schistocerca gregaria (Forskal), from our crowded culture were mounted ventral surface uppermost. The apodeme of the femoral chordotonal organ (FCO) was cxposed by dissecting away cuticle on the anterior face of the femur, proximal to the heavily sclerotized, black semilunar process. Full details of the dissection and a drawing of the anatomy of the FCO are given in Burrows (1987a). The femorotibial joint was set at $80^{\circ}$ and the apodeme of the FCO grasped between the tips of forceps attached to a vibrator (Ling; Altec type 101), so that it could be moved in waveforms of controlled frequency and amplitude. A stretch applied to the apodeme corresponds to a stimulus that would normally occur during flexion of the femorotibial joint, and a movement that allows the FCO to relax corresponds to extension. A movement of the apodeme will be described as either a flexion or extension, reflecting the way it would normally be activated in the leg. A $500 \mu \mathrm{m}$ movement of the apodeme corresponds to a $40^{\circ}$ movement of the femorotibial joint, between joint angles of $20^{\circ}$ and $130^{\circ}$ (Field and Burrows, 1982). Repetitive movements of the FCO were applied only in the range of stepping frequencies $(1-9 \mathrm{~Hz}$ ) used by the locust (Burns, 1974). Spikes of the FCO afferents were recorded extracellularly by a pair of hook electrodes made from $100-\mu \mathrm{m}$-diameter silver wire and placed on N5b1 (Campbell, 1961), just proximal to the point at which the FCO nerve joins.

The meso- and metathoracic ganglia were exposed and stabilized on a wax-coated steel platform and the thoracic cavity perfused with a constant flow of saline. The sheath of the metathoracic ganglion was treated with a $0.1 \%(\mathrm{wt} / \mathrm{vol})$ solution of protease (Sigma type XIV) before recording began. The neuropilar processes of nonspiking interneurons and the cell bodies of motor neurons and interneurons were impaled with microelectrodes filled with $2 \mathrm{M}$ potassium acetate that had DC resistances of $50-80 \mathrm{M} \Omega$ or, in some cxperiments, with $0.1 \mathrm{M}$ hexaminc cobaltic chloride. Nonspiking interneurons were identified according to established criteria (see Burrows and Siegler, 1978, for full details). The interneurons did not spike during imposed movements of the FCO apodeme or when current was injected through the recording electrode. Some could be characterized by their effects on known leg motor neurons when depolarized with injected current. Identification was completed by intracellular staining with cobalt (Brogan and Pitman, 1981). FCO afferents were stained by backfilling with cobalt (see Burrows, 1987a, for full details). Stained neurons were drawn from whole-mounts of the metathoracic ganglion and from serial transverse sections cut at $10 \mu \mathrm{m}$. Motor neurons were identified by correlating their intracellularly recorded spikes with potentials recorded extracellularly from a particular muscle, using a pair of $50 \mu \mathrm{m}$ steel wires insulated except at their tips.

All recordings were stored on magnetic tape for later display on a Gould ES1000 recorder, or on an X-Y plotter, after capture with a digital storage oscilloscope. The physiological results are based on 36 successful recordings from an interneuron and a motor neuron in 20 locusts.

\section{Results}

Nonspiking interneurons can alter proprioceptive reflexes The proprioceptive signals provided by the FCO during movements of the tibia about the femur evoke reflex responses in certain muscles of the ipsilateral hindleg. Two types of reflexes in response to an imposed movement can be distinguished. The first consists of resistance reflexes in which an imposed movement is met by an increase in the force generated by an appropriate muscle. In the second, interjoint reflexes make adjustments in other joints of the leg to compensate for movements of the femorotibial joint.

An imposed flexion of the FCO evokes spikes in the slow extensor tibiae motor neuron, and an imposed extension excites flexor tibiae motor neurons (Fig. 1A). Resistance reflexes, therefore, result: the imposed movement is resisted by an active contraction of the appropriate muscle. During the resistance reflex to an imposed flexion of the $\mathrm{FCO}$, a nonspiking interneuron is depolarized at the time when the slow extensor motor neuron spikes (Fig. 1A). This nonspiking interneuron excites the motor neuron when depolarized by a pulse of current injected 

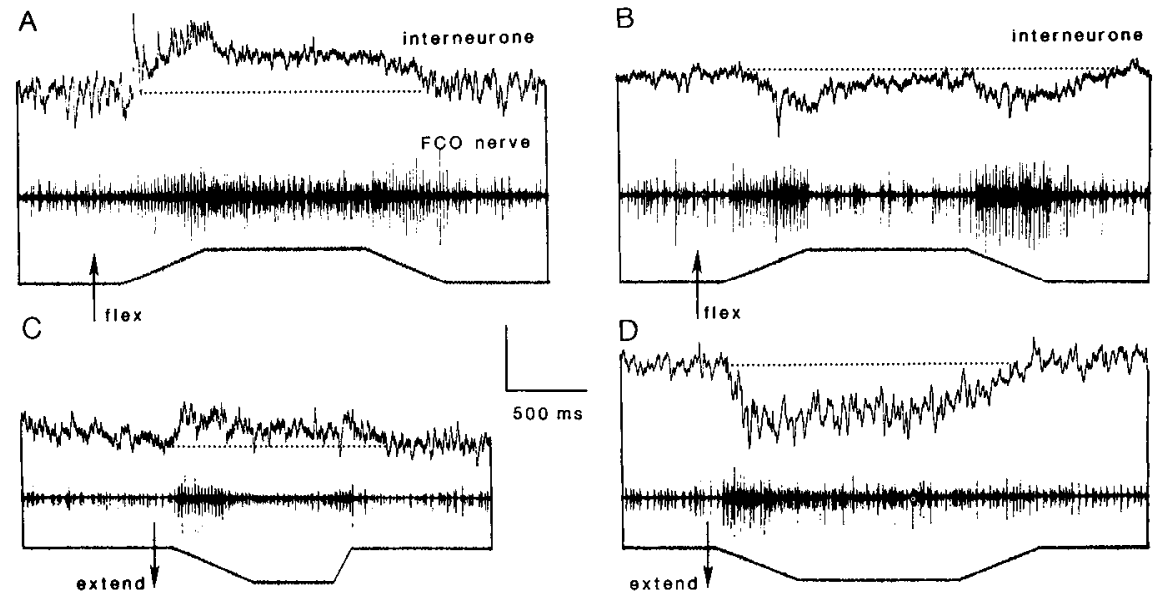

Figure 2. Movements of the FCO apodeme excite or inhibit nonspiking local interneurons. $A, \mathrm{~A}$ movement corresponding to a $40^{\circ}$ flexion of the femorotibial joint excites many afferents (bottom trace) and evokes a phasic burst of depolarizing potentials in the interneuron (top trace). During the maintained flexion, afferents spike tonically and the interneuron is also tonically depolarized. $B$, A similar stimulus in another locust evokes a burst of hyperpolarizing potentials in a second interneuron at both the flexion and extension phases of the movement. $C$, An extension movement depolarizes a third interneuron. $D$, A similar movement hyperpolarizes a fourth interneuron. Dotted lines in this and subsequent figures indicate the average membrane potential before the imposed movement. Each record is from a different locust. Calibration: vertical, $4 \mathrm{mV}$ (interneurons $A, B, D) ; 2 \mathrm{mV}(C)$; movement, $50^{\circ}$

through the recording electrode. If this interneuron is hyperpolarized by a steady current, the response of the extensor motor neuron to the same FCO stimulus is reduced (Fig. $1 B$ ). In the example shown in Figure 1, the number of spikes is reduced from 5-7 at each flexion to 1 , but the reduction is graded and depends on the current that is injected.

An imposed flexion of the FCO also excites the levator tarsi motor neuron so that the tarsus is reflexly levated (Fig. 1C). At the same time, another nonspiking interneuron is depolarized by the imposed movement. This nonspiking interneuron excites the levator motor neuron when depolarized with a pulse of current. If this interneuron is depolarized by a steady current, the response of the levator motor neuron to the same flexion movement of the FCO is increased (Fig. 1D). Again, the effect is graded, depending on the current that is injected.

These observations indicate an important role for the nonspiking interneurons in controlling these reflexes. First, the 2 nonspiking interneurons in these examples are both depolarized by signals from flexion-sensitive afferents of the FCO. Second, they make excitatory connections with particular leg motor neurons. Third, their causal role in the reflexes is shown by their ability to alter the gain of a reflex when their membrane potential is manipulated. To understand the role of these interneurons, the pathways by which they receive the afferent signals need to be elucidated.

\section{Effects of $F C O$ inputs on nonspiking interneurons}

A movement imposed on the FCO apodeme excites some nonspiking interneurons and inhibits others. Individual interneurons have characteristic responses to a particular movement, as is illustrated in Figure 2. The first interneuron is depolarized both phasically and tonically by a flexion movement (Fig. $2 A$ ). During the flexion it receives a barrage of depolarizing potentials, and while in a more flexed position, a tonic depolarization is sustained. During extension, the membrane potential returns to its original level and hyperpolarizing potentials resume. A second interneuron is hyperpolarized phasically both during a flexion and an extension movement (Fig. 2B). A third interneuron is depolarized phasically by both an extension and a flexion (Fig. 2C). A fourth interneuron is hyperpolarized by extension and remains hyperpolarized until the apodeme is moved back to its starting position (Fig. 2D). Interneurons 1 and 4 are thus directionally sensitive, but interneurons 2 and 3 are not.

\section{Excitation is mediated by direct afferent inputs}

During flexion, the nonspiking interneuron shown in Figure $3 \mathrm{~A}$ receives depolarizing synaptic potentials that are associated with groups of spikes from FCO afferents. During extension, different afferents, but with spikes of similar amplitude, are excited and are associated with depolarizing potentials in a flexor tibiae motor neuron (Fig. 3A). They are not, however, associated with a response in the interneuron. On an expanded time scale, each group of spikes in flexion-sensitive afferents is seen to precede each depolarization in the interneuron (Fig. 3B). Triggering a signal averager with one of these spikes, selected by its amplitude, reveals a linked depolarizing potential in the interneuron (Fig. 3C). Electrical stimulation of the FCO afferent nerve also evokes a depolarizing synaptic potential with a constant latency and without failures in the interneuron. The latency is the same as that to an EPSP evoked simultaneously in a flexor tibiae motor neuron (Fig. 3D). The EPSP in the flexor motor neuron has been shown to be evoked by direct connections from FCO afferents and to have a central latency of $1.5 \mathrm{msec}$ (Burrows, 1987a).

Hyperpolarizing the interneuron with a steady current while stimulating the FCO nerve electrically enhances the amplitude of the evoked potential (Fig. $3 E$ ). The evidence, therefore, indicates that some of the flexion-sensitive afferents make direct, chemically mediated connections with this nonspiking interneuron. Similar evidence led to the same conclusion for all nonspiking interneurons that received EPSPs during flexion or extension of the FCO.

Inhibition is mediated by interposed spiking local interneurons

No FCO afferents were found to make direct inhibitory connections with nonspiking interneurons. Nevertheless, some nonspiking interneurons are inhibited by movements of the FCO, 

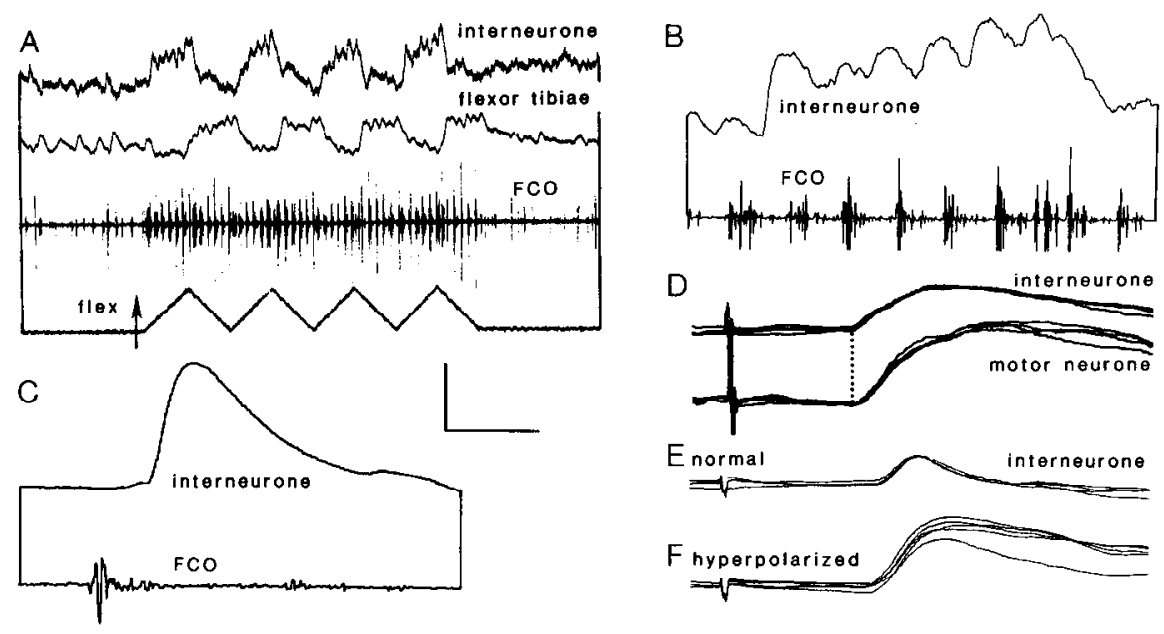

Figure 3. FCO afferents directly excite a nonspiking interneuron. A, An imposed flexion evokes a discharge of FCO afferent spikes (bottom trace) and a burst of depolarizing potentials in the interneuron (top trace). An imposed extension evokes spikes in different afferents and a burst of depolarizing potentials in a flexor tibiae motor neuron (middle trace). $B$, During flexion, bursts of afferent spikes precede each wave of depolarization in the interneuron. The last burst of spikes precedes the first depolarization in the flexor motor neuron. $C$, Signal averages (128 sweeps) triggered from a particular amplitude of afferent spike selected by a window circuit show a linked EPSP in the interneuron. $D$, EPSPs occur with the same latency in both the interneuron and a flexor tibiae motor neuron when the FCO nerve is stimulated electrically with $0.3 \mathrm{msec}$ pulses. Presumably the stimulus excites several afferents simultaneously, irrespective of their directional sensitivity. $E, F$, Hyperpolarizing the interneuron increases the amplitude of the EPSP evoked by electrical stimulation from $2.4(E)$ to $6 \mathrm{mV}(F)$. Records $A$ and $B$ are from one, $C$ and $D$ from a second, and $E$ and $F$ from a third locust. The recording/stimulating electrodes were $28 \mathrm{~mm}$ from the ganglion. Calibration: vertical, nonspiking interneuron, $2 \mathrm{mV}(A) ; 1 \mathrm{mV}(B) ; 0.9 \mathrm{mV}(D)$; motor neuron, $10 \mathrm{mV}(A) ; 1 \mathrm{mV}(D) 6 \mathrm{mV}(E, F)$; movement, 50\%; horizontal, $250 \mathrm{msec}(A) ; 35.5 \mathrm{msec}(B) ; 20$ $\mathrm{msec}(C) ; 8.2 \mathrm{msec}(D) ; 7 \mathrm{msec}(E, F)$.

and this inhibition involves the action of spiking local interneurons. For example, one spiking local interneuron makes an inhibitory connection with the nonspiking interneuron shown in Figure 4. The latency of $0.6 \mathrm{msec}$ between the peak of the spike and the IPSP suggests a direct connection (Fig. 4A) (see Burrows, $1987 \mathrm{~b}$, for further details). When the FCO signals an extension of the tibia, the spiking interneuron is excited and spikes rapidly, and, at the same time, the nonspiking interneuron is inhibited (Fig. $4 B$ ). Repeated extensions of the tibia produce correlated changes in both neurons (Fig. $4 C$ ). When the spiking

A
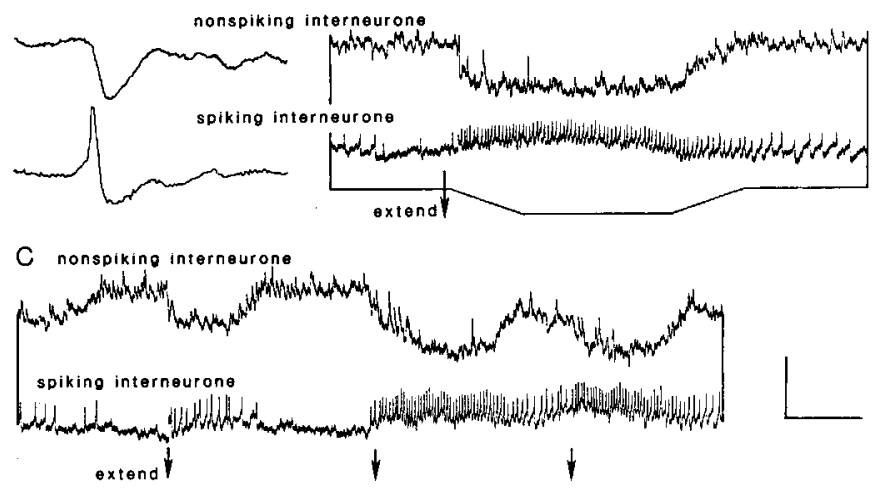

Figure 4. The inhibitory effects of FCO afferents on a nonspiking interneuron are mediated by interposed spiking local interneurons. $A$, A signal average (256 sweeps) shows that spikes in a spiking interneuron are followed by an IPSP in a nonspiking interneuron. $B$, An extension movement evokes a sustained burst of spikes in the spiking interneuron (middle trace) and a sustaincd hypcrpolarization of the nonspiking interneuron (top trace). $C$, Repeated extensions of the tibia evoke reciprocal responses in the 2 interneurons. Calibration: vertical, nonspiking interneuron, $10 \mathrm{mV}$; spiking interneuron, $5 \mathrm{mV}(B) ; 10 \mathrm{mV}(C)$; movement, $50^{\circ}$; horizontal, $28 \mathrm{msec}(A) ; 500 \mathrm{msec}(B, C)$. interneuron spikes at low frequency, the hyperpolarization of the nonspiking interneuron is small (see first arrow in Fig. 4C), and when it spikes faster, the hyperpolarization of the nonspiking interneuron is correspondingly enhanced (second arrow in Fig. 4C). Hyperpolarization of the impaled spiking interneuron to suppress its spikes reduces, but does not abolish, the inhibition of the nonspiking interneuron, demonstrating the convergence of inhibitory inputs from several spiking interneurons. The inhibition of the nonspiking interneuron thus results from disynaptic pathways in which FCO afferents directly excite spiking local interneurons (Burrows, 1987a), which then inhibit the nonspiking interneuron.

Anatomy of the interneurons is consistent with these pathways Branches of the $\mathrm{FCO}$ afferents and nonspiking interneurons must overlap in the neuropil if direct connections between them are to exist. Nonspiking interncurons that are excited by afferents of the chordotonal organ have a variety of shapes, with cell bodies in different locations in the metathoracic ganglion (Watkins et al., 1985). One interneuron that is excited by extension of the FCO has a medial and ventral cell body with extensive branches in one-half of the ganglion (Fig. $5 \mathrm{~A}$ ). When sections of the ganglion containing this stained interneuron are compared with those from a ganglion containing stained FCO afferents, considerable overlap is apparent in their branches (Fig. 5, $B$, $C)$. In the sections shown, branches both of the afferents and the interneuron occur in neuropil lateral to the longitudinal median ventral tract (MVT).

\section{Demonstrated pathways explain the effects produced by the FCO}

Nonspiking interneurons sensitive to movement of the FCO can receive (1) direct excitation from somc of the afferents, (2) inhibition from spiking local interneurons that are themselves 


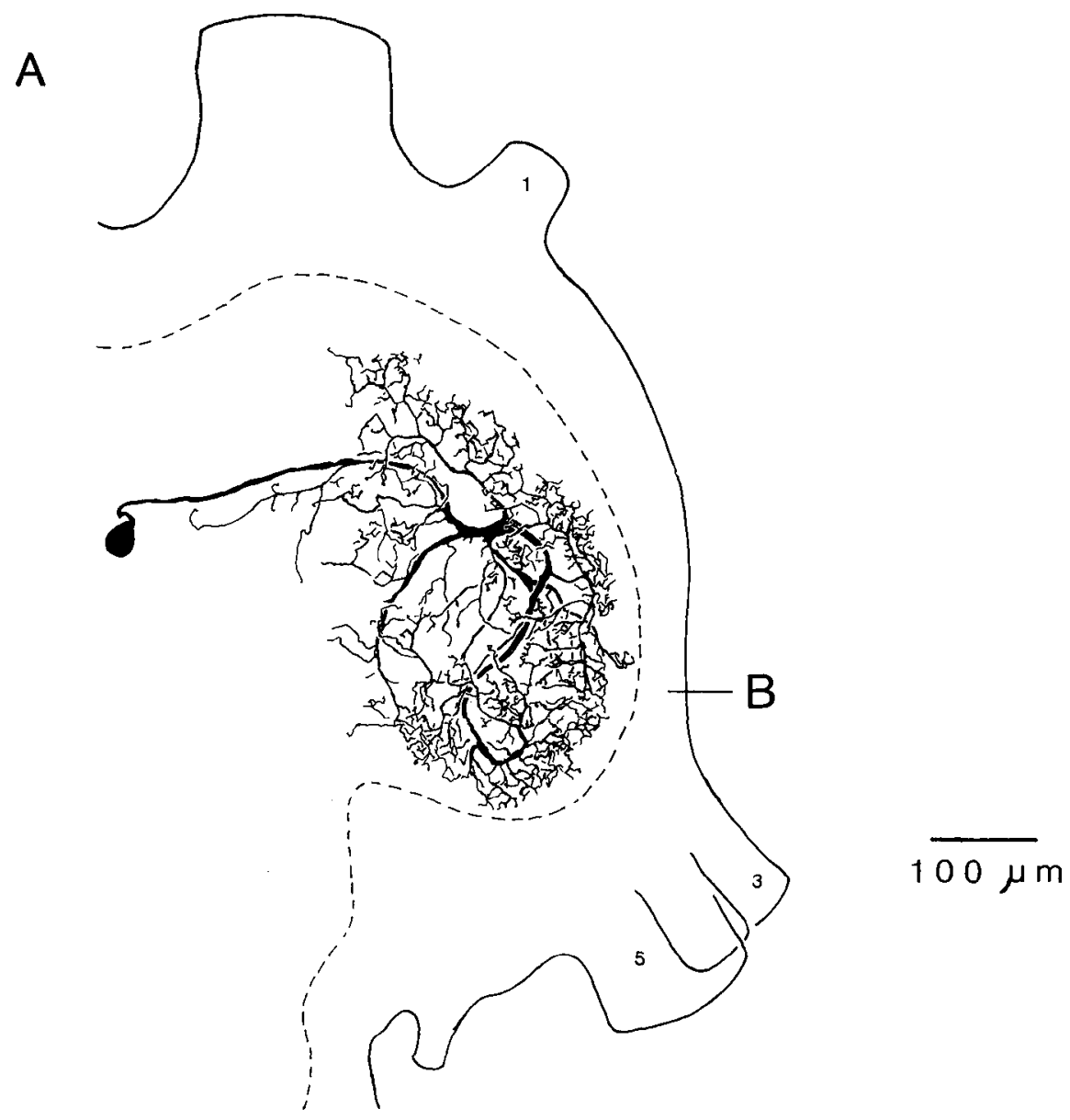

$B$ interneurone

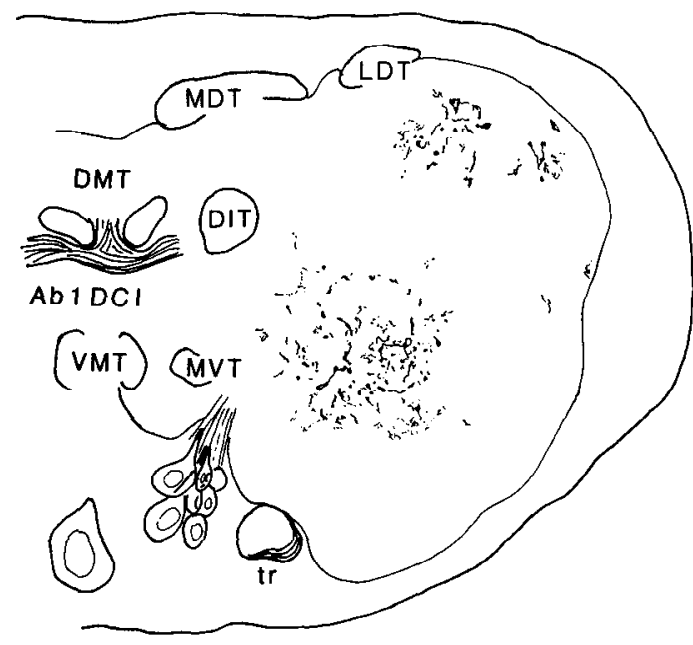

C FCO afferents

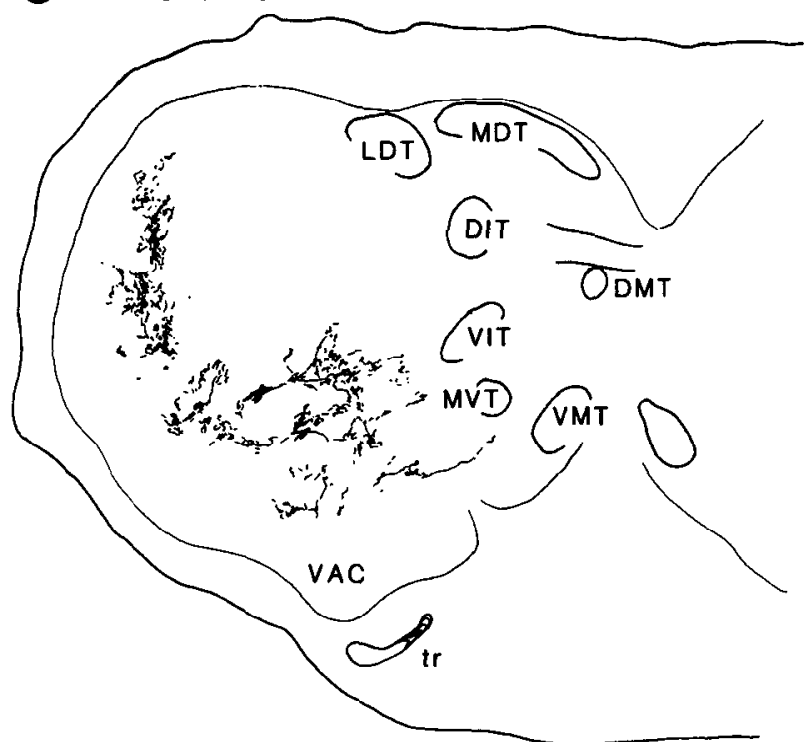

Figure 5. Afferents from the FCO and the branches of a nonspiking interneuron they excite project to the same regions of neuropil. $A$, An interneuron excited by extension has a ventral cell body and extensive branches in one-half of the ganglion. It is drawn in a whole-mount of the metathoracic ganglion viewed dorsally and with anterior at the top. Nerves $l$ and 3-5 are numbered, and the dashed line indicates the approximate boundary of the neuropil. $B, C$, Transverse sections through the interneuron $(B)$ and at approximately the same plane through a ganglion in which chordotonal afferents were stained $(C)$ show branches of both in the same lateral region of neuropil. Abbreviations, based on Tyrer and Gregory (1982): $A b 1$, Abdominal neuromere 1; $D C I$, dorsal commissure I; $D I T$, dorsal intermediate tract; $D M T$, dorsal median tract; $L D T$, lateral dorsal tract; $M D T$, median dorsal tract; $M V T$, median ventral tract; $t r$, trachea; $V A C$, ventral association center; $V I T$, ventral intermediate tract; $V M T$, ventral median tract. 

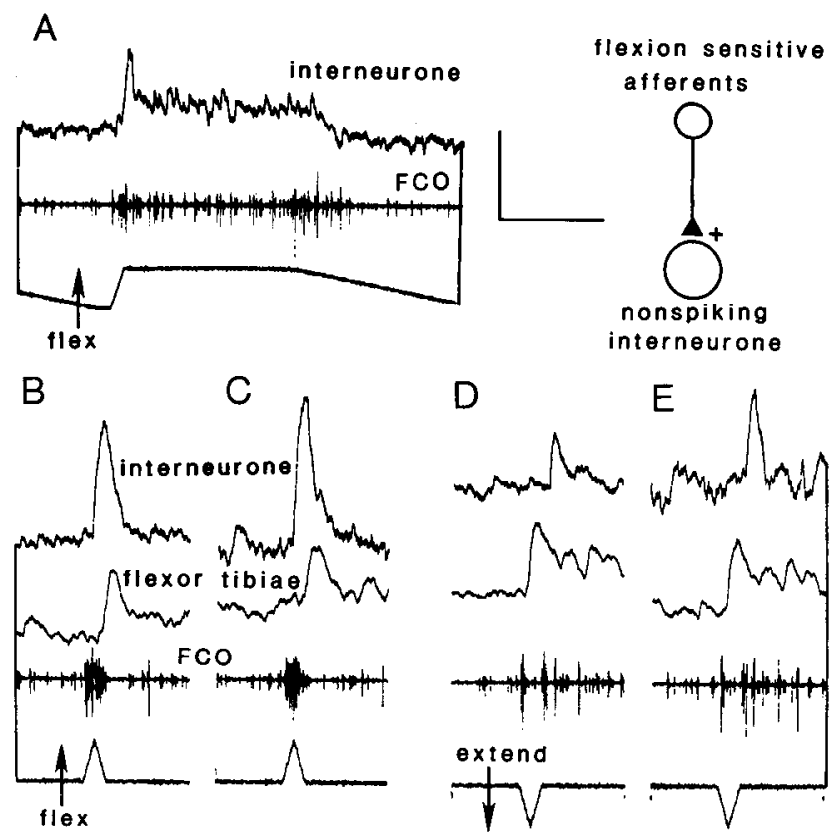

Figure 6. A nonspiking interneuron whose responses to FCO movements are explained by excitatory inputs (see inset). $A$, A flexion of the FCO evokes a depolarization of the interneuron with both phasic and tonic components. When the FCO is extended, the interneuron repolarizes. $B-E$, The interneuron (top trace) is recorded with a flexor tibiae motor neuron (middle trace). $B$. The interneuron is depolarized by a rapid flexion and the motor neuron by a rapid extension. $C$, Hyperpolarizing the interneuron with a steady current enhances its response to an imposed flexion. $D$, An extension movement that precedes flexion excites the motor neuron but has no effect on the interneuron. The latter is, however, depolarized by the following flexion movement. $E$, The response of the interneuron to flexion is enhanced by an applied hyperpolarization, but no response to extension is revealed. All recordings are from one locust and all movements start from the same set position of the FCO apodeme, equivalent to a femorotibial angle of $80^{\circ}$. Calibration: vertical, interneuron, $4 \mathrm{mV}$; motor neuron, $10 \mathrm{mV}$; movement, $50^{\circ}$; horizontal, $500 \mathrm{msec}(A) ; 250 \mathrm{msec}(B-E)$.

excited directly by some of the afferents, or (3) a combination of the 2, where excitation and inhibition are each associated with a different direction of movement. Additional pathways involving more complex interactions among the local interneurons provide further excitation or inhibition. The possible contribution of intersegmental interneurons is not explored in this paper.

Excitation. A flexion of the FCO excites the interneuron shown in Figure 6. This movement evokes a response with an initial phasic component followed by a tonic one, which is maintained for as long as the new flexed position is held (Fig. 6A). An extension, by contrast, is simply followed by a repolarization of the interneuron. A rapid flexion and extension movement evokes a depolarization of the interneuron upon flexion and a depolarization of a flexor tibiae motor interneuron upon extension (Fig. 6B). If the extension movement precedes the flexion, however, it can more clearly be seen to have no effect on the interneuron, but to evoke a depolarization of the motor neuron (Fig. 6D). The amplitude of the depolarization evoked by a flexion is enhanced when the membrane of the interneuron is hyperpolarized, suggesting that EPSPs underlie the response (Fig. $6, C, E$ ). No IPSPs that are associated with the stimulus are revealed; therefore, the background IPSPS visible at resting potential are not linked to FCO afferent input. Moreover, stimulation of the FCO nerve evokes only EPSPs in this interneuron, in contrast to the IPSPs evoked by a similar regime of stimulation in other interneurons (see Fig. 7C).

The responses of this interneuron can thus be explained by direct excitatory inputs from flexion-sensitive afferents. The repolarizations during extension movements result from the decreased activity of these afferents and not from inhibitory inputs from spiking local interneurons excited by extension-sensitive afferents (see inset in Fig. 6).

Inhibition. Other interneurons apparently receive only an inhibitory input as a result of FCO stimulation. In the absence of a stimulus, the interneuron in Figure 7 receives a barrage of synaptic potentials, the majority of which are hyperpolarizing (Fig. $7 A$ ). When the FCO is Hexed, the frequency of these potentials decreases, the membrane depolarizes, and a few clear hypcrpolarizations are seen (Fig. $7 A$ ). When the FCO is returned to its original position, the high-frequency synaptic potentials resume, and the membrane potential of the interneuron is transiently hyperpolarized below the level at the onset of the stimulus (Fig. 7A). A hyperpolarizing current applied through the recording electrode can reverse the hyperpolarizing potentials, indicating that they are IPSPs. During repetitive movements of the $\mathrm{FCO}$, the interneuron depolarizes during flexion and is hyperpolarized during extension by an inhibitory input, so that its membrane potential oscillates about its "resting" level (Fig. $7 B$ ). This contrasts with the response of a flexor tibiae motor neuron recorded simultaneously, which receives an excitatory input during extension, but no inhibitory input during flexion, so that its membrane potential oscillates above its "resting" level (Fig. $7 B$ ). When the FCO nerve is stimulated electrically, IPSPs are evoked in the nonspiking interneurons, occurring with a latency approximately $3 \mathrm{mscc}$ longer than that to the EPSP evoked in the flexor tibiae motor neuron (Fig. 7C). A sufficient explanation of the responses of this interneuron is that it receives inhibitory inputs from spiking local interneurons which are excited during extension (see insert in Fig. 7). During flexion, the frequency of spikes in these interneurons decreases, and this, in turn, releases the nonspiking interneuron from its inhibition.

The role of spiking local interneurons in mediating inhibition and disinhibition of a nonspiking interneuron is shown in Figure 8. Each of the spikes in a spiking interneuron is followed, after a consistent latency of $0.6 \mathrm{msec}$, by an IPSP in the nonspiking interneuron (Fig. 8A), a connection confirmed by signal averaging (Fig. $8 B$ ). The spiking interneuron spikes tonically when no stimulus is applied to the FCO, so that the nonspiking interneuron is held hyperpolarized. When the $\mathrm{FCO}$ is flexed, these tonic spikes are suppressed, so that the inhibitory input to the nonspiking interneuron is withdrawn (Fig. 8C). When the FCO is extended again, the spikes and their inhibitory effects resume. During rapid, repetitive movements of the $\mathrm{FCO}$, the membrane potential of the spiking interneuron is clearly modulated by each cycle of movement, but it does not spike (Fig. 8D). The nonspiking interneuron is therefore allowed to depolarize throughout the period of stimulation, and shows no response to individual cycles of movement, in contrast to the interneuron in Figure 7 . The effect of the movement on this spiking interneuron, in contrast to that on the putative spiking interneurons in Figure 7 , is thus purely inhibitory.

The response of the nonspiking interneuron can therefore be explained by a pathway involving at least 2 spiking interneurons in series. One spiking interneuron make a direct inhibitory connection with the nonspiking interneuron, but is not itself excited directly by the FCO afferents. Instead, another spiking interneuron is involved: it reccives inputs from flcxion-sensitive af- 
A
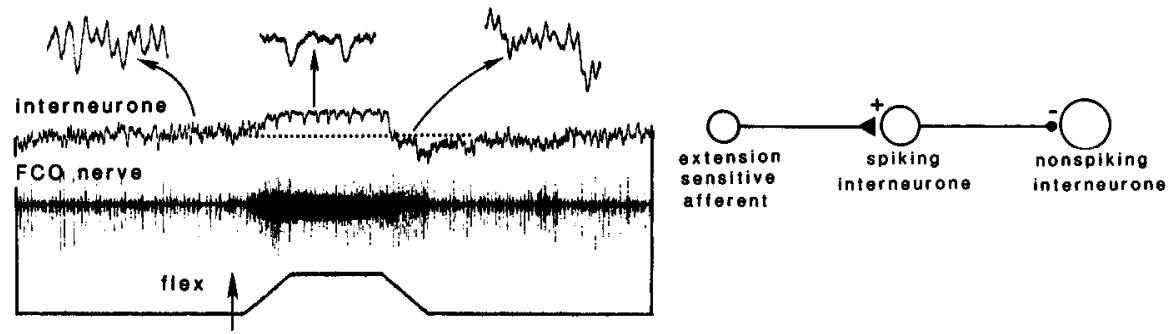

B
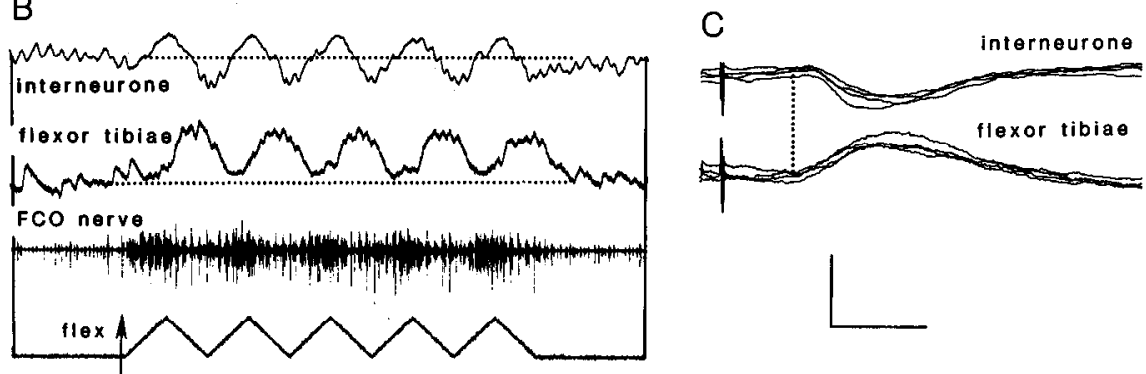

Figure 7. A nonspiking interneuron whose responses to FCO movements are explained by inhibitory inputs (see inset). $A$, With no movement of the FCO, the interneuron receives a constant barrage of IPSPs. An imposed flexion evokes a depolarization of the interneuron due to a reduction in the frequency of these IPSPs. When the FCO is extended, the IPSPs resume. The changes in frequency of the IPSPs are shown on expanded scales in the insets (arrows). B. The interneuron is now recorded with a flexor tibiae motor neuron. Repetitive movements of the FCO evoke changes in membrane potential of both neurons that follow each cycle of the stimulus. IPSPs are evoked in the interneuron during extension and decrease in frequency during flexion, so that the membrane potential oscillates about the resting level (dotted line). In contrast, the motor neuron is depolarized during extension and its membrane potential oscillates above its resting potential. $C$, Electrical stimulation of the FCO nerve evokes an IPSP in the nonspiking interneuron that has a longer latency than an EPSP in the flexor tibiae motor neuron. All recordings are from the same locust. Calibration: vertical, interneuron, $10 \mathrm{mV}(A, B) ; 4 \mathrm{mV}(A$, inset); $2.5 \mathrm{mV}(C) ; 4 \mathrm{mV}$ (motor neuron $B) ; 2.5 \mathrm{mV}(C) ; \mathrm{movement}$, $50^{\circ}$; horizontal, $1 \mathrm{sec}(A) ; 250 \mathrm{msec}(B) ; 14.2 \mathrm{msec}(C)$.

ferents and then inhibits the other spiking interneuron. Disinhibition of the nonspiking interneuron will result whenever Hexion is signaled by the FCO.

Inhibition and excitation. The interneuron shown in Figure 9 receives both excitation and inhibition during movements of the FCO. During flexion, the interneuron is depolarized by groups of potentials that correspond to the groups of spikes in the flexion-sensitive FCO afferents (Fig. 9A). These depolarizing potentials are increased in amplitude by the application of a steady hyperpolarizing current, indicating that they are EPSPS (Fig. 9B). By contrast, during extension the interneuron is hyperpolarized (Fig. 9C) by potentials that can be reversed in polarity by an applied hyperpolarization, indicating that they are IPSPs. Electrical stimulation of the FCO nerve evokes an
EPSP, and, with stimuli of greater intensity, an IPSP with a longer latency that curtails the falling phase of the EPSP (Fig. 9C). The excitation of this nonspiking interneuron can thus be explained by direct connections of flexion-sensitive afferents, and the inhibition by intcrposed spiking intcrncurons excited by extension-sensitive afferents (see inset in Fig. 9).

\section{Discussion}

\section{Excitation and inhibition of nonspiking local interneurons}

Nonspiking interneurons are essential elements in the integration of a proprioceptive signal from the femoral chordotonal organ into reflex contractions of the leg muscles. Their importance is emphasized by the observation that experimental manipulation of the membrane potential of a single interneuron
A

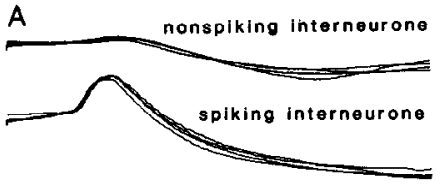

B

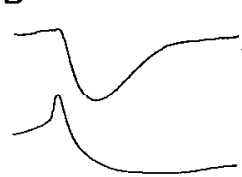

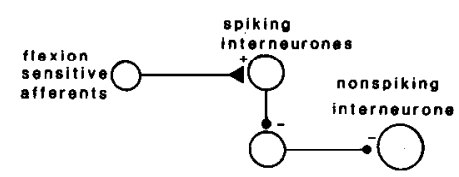
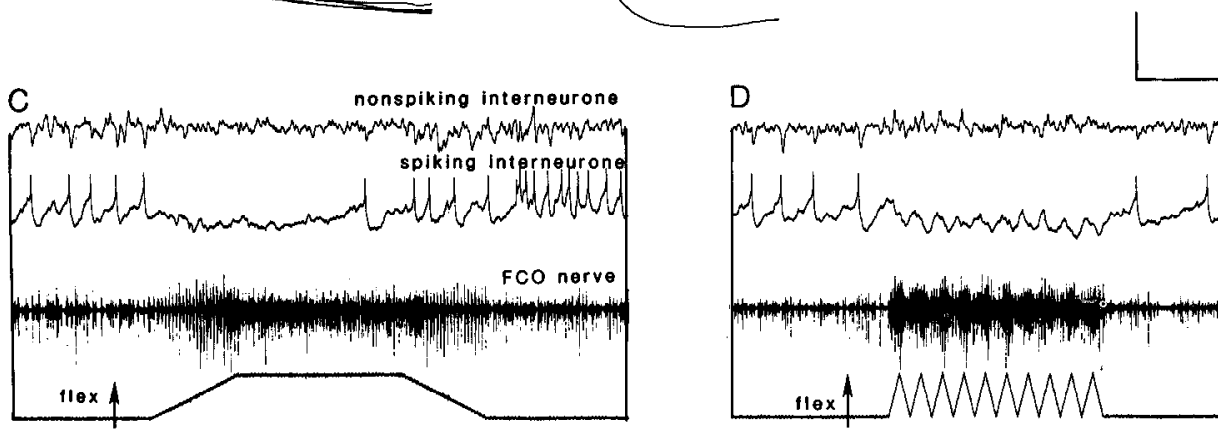

D

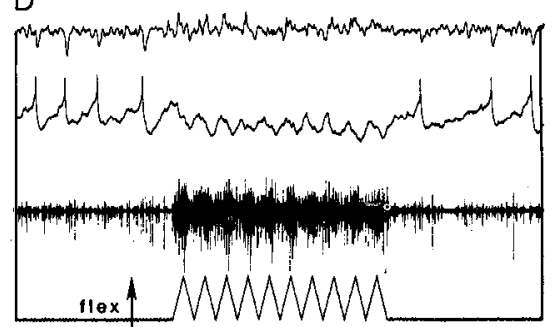

Figure 8. A nonspiking interneuron whose responses to FCO movements are explained by a cessation of inhibitory inputs from a spiking local interneuron (see inset). $A$, Spikes in the spiking interneuron are followed after a short and consistent latency by IPSPs in the nonspiking interneuron. $B$, The inhibitory connection is confirmed by signal averaging, 128 sweeps. $C$, A flexion inhibits the spikes in the spiking interneuron and the IPSPs in the nonspiking interneuron cease. $D$, During repetitive movements, the spiking interneuron does not spike, so that the nonspiking interneuron fails to follow each cycle of the movement. Calibration: vertical, nonspiking interneuron, $2.5 \mathrm{mV}(A) ; 10$ $\mathrm{mV}(C, D)$; spiking interneuron, $5 \mathrm{mV}$ $(A)$; $10 \mathrm{mV}(C, D)$; movement, $50^{\circ}$; horizontal, $4 \mathrm{msec}(A) ; 20 \mathrm{msec}(B) ; 500$ $\operatorname{msec}(C, D)$. 


\section{A}
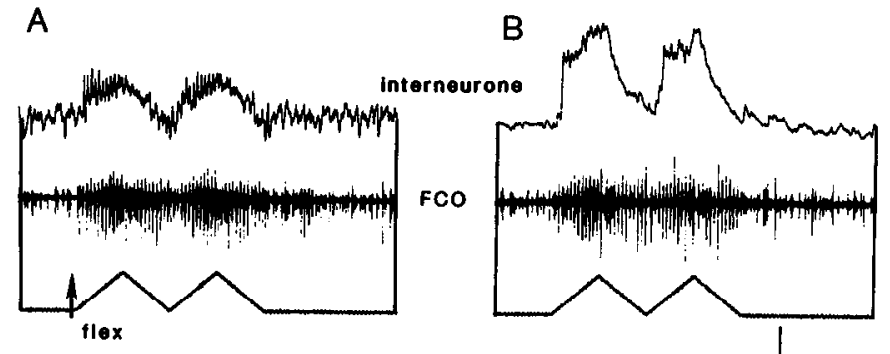

C

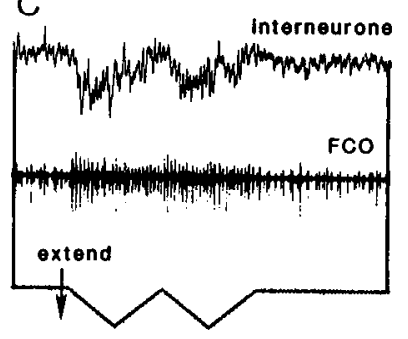

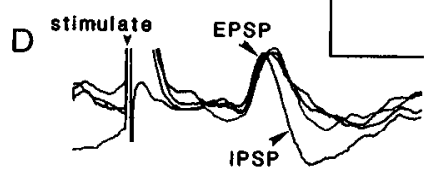

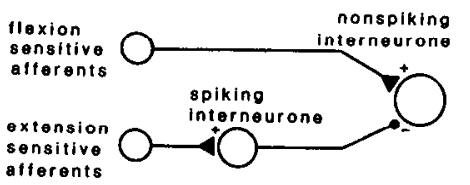

Figure 9. A nonspiking interneuron whose responses to FCO movements are explained by an excitatory input during flexion and an inhibitory input during extension (sce inset). $A$, Imposed flexion movements evoke EPSPs in the interneuron (top trace). $B$, Hyperpolarizing the interneuron with a steady current enhances the amplitude of these evoked EPSPs. $C$, Imposed extension movements evoke IPSPs in the interneuron. $D$, Electrical stimulation of the FCO nerve evokes an EPSP with a short latency, and, with stronger stimulus intensity, an IPSP with a longer latency that curtails the EPSP. Calibration: vertical, $4 \mathrm{mV}(A-$ $C) ; 2 \mathrm{mV}(D)$; movement, $50^{\circ}$; horizontal, $500 \mathrm{msec}(A-C) ; 11 \mathrm{msec}$ (D).

can alter the response of the participating motor neurons to the proprioceptive signal. Some afferents can directly excite the nonspiking interneurons, but can inhibit them only through the action of interposed interneurons. The evidence for direct afferent connections is that afferent spikes evoked by movement of the receptor apodeme, or by electrical stimulation of the afferent nerve, are consistently followed by EPSPs at a constant, short latency in particular interncurons. We have not addressed the issue of whether all afferents that provide information about different aspects of a particular movement, such as velocity or acceleration, make the same connections with a given interneuron. The EPSPs in the nonspiking interneurons occur with the same latency as those that are also evoked in flexor tibiae motor neurons. The connection of some FCO afferents with flexor tibiae motor neurons has been shown to be direct by simultaneous intracellular recording from an afferent cell body in the FCO and from the cell body of a flexor motor neuron in the metathoracic ganglion (Burrows, 1987a). With this experimental arrangement, current could be injected into the afferent to evoke spikes that are followed 1:1 by EPSPs in the motor neuron. The central latency of these EPSPs is $1.5 \mathrm{msec}$. This value consists of the time for conduction of the afferent spike in the ganglion to the synaptic sites and for synaptic transmission, and suggests that no more than one synapse is involved in the pathway. A reasonable interpretation of these observations is that the connection is direct, and that, therefore, whatever conclusion is drawn about the afferent connections with motor neurons should logically also apply to the connections demonstrated here with the nonspiking interneurons. The anatomical observations of overlap of the branches of the participating neurons provide further support for this conclusion. A similar conclusion has been reached for a second class of afferents, the exteroceptors

on a leg, which, on both anatomical and physiological grounds, appear to excite directly the nonspiking interneurons (Laurent and Burrows, 1988).

Afferent spikes cannot, however, be correlated with the IPSPs evoked in the interneurons. Electrical stimulation of the afferent nerve evokes IPSPs that have a longer latency than do EPSPs, suggesting a pathway involving interposed spiking interneurons. Some spiking local interneurons that have cell bodies at the ventral midline of the ganglion (Siegler and Burrows, 1984) receive direct inputs from afferents of the chordotonal organ (Burrows, 1987a) and make direct inhibitory connections with nonspiking interneurons (Burrows, 1987b). Here, these connections are shown to be essential in shaping the response of a nonspiking interneuron to an input from the FCO. The result is that a particular proprioceptive afferent may directly excite some nonspiking local interneurons and at the same time inhibit others by a disynaptic pathway involving spiking local interneurons. More complex pathways mediating inhibition may also exist in parallel. For example, nonspiking interneurons make inhibitory connections with one another (Burrows, 1979), so that the disinhibition in Figure 7 could be aided by the withdrawal of afferent inputs from a nonspiking interneuron with an inhibitory connection to the impaled interneuron. Similarly, inhibition of a nonspiking interneuron could be aided by parallel inputs from other nonspiking interneurons or even by intersegmental interneurons. Unraveling these more convoluted pathways has not been our objective, but this does not imply that their contribution can be neglected.

\section{Proprioceptive reflex pathways}

The connections demonstrated here can now be combined with others already known to reconstruct the pathways used for proprioceptive reflexes (Fig. 10). Consider an imposed extension movement of the FCO that excites a certain population of afferents. The imposed movement sets up a resistance reflex in which flexor tibiae motor neurons are excited and extensor motor neurons inhibited. An interjoint reflex is also set up in which depressor tarsi motor neurons are excited and the levator tarsi inhibited.

The flexor tibiae motor neurons are excited by the proprioceptive afferents either directly or by nonspiking interneurons acting in parallel. By contrast, the inhibition of extensor tibiae motor neurons is always mediated by local interneurons, with a possible, but as-yet unknown, contribution from other types of interneurons. The afferents excite both nonspiking and spiking local interneurons, which make direct inhibitory connections with the extensor motor neurons (Burrows and Siegler, 1978, 1982). The pathways are made more complex by the interactions among the local interneurons themselves. Nonspiking interneurons can be inhibited by other nonspiking and by spiking local interneurons. For example, a nonspiking interneuron that excites the extensor motor neurons can be inhibited by spiking and nonspiking interneurons that receive direct inputs from the afferents. The effect will be to reduce the excitation impinging on the extensor motor neurons.

Similar pathways control the motor neurons of adjacent joints of the leg. Depressor tarsi motor neurons are excited by nonspiking interneurons that receive direct afferent inputs. The levator tarsi motor neuron is inhibited by nonspiking and spiking local interneurons and by the inhibition of nonspiking interneurons with excitatory connections.

Therefore, in any particular reflex movement, parallel path- 


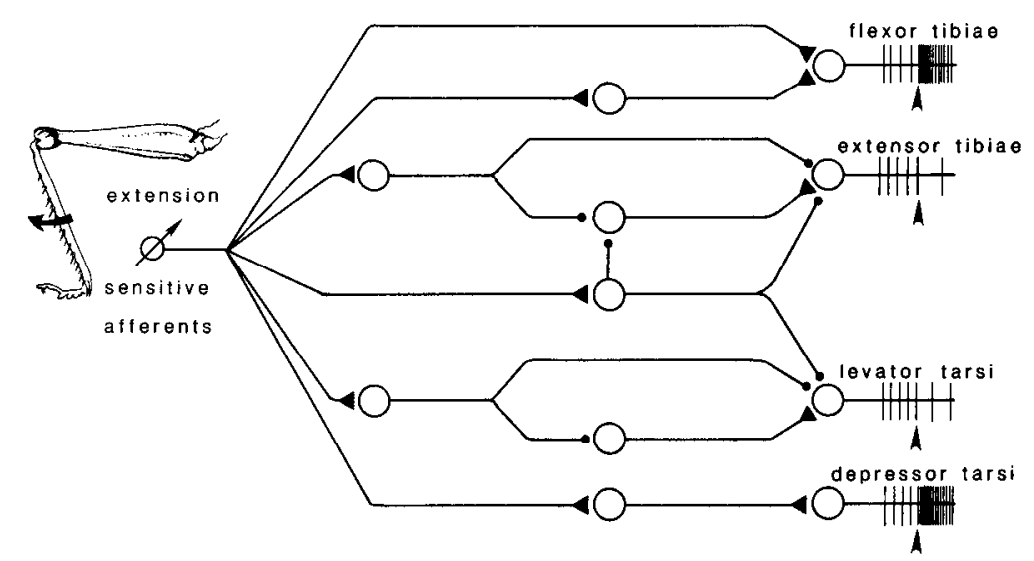

Figure 10. Pathways mediating a resistance reflex of leg muscles initiated by sensory signals from the femoral chordotonal organ. This is not a circuit diagram, but indicates the flow of information along known pathways involving identified neurons. Excitatory synapses are indicated by triangles, inhibitory ones by circles.

ways predominate. Nevertheless, individual nonspiking interneurons play a crucial role. Hyperpolarizing an interneuron reduces the efficacy of a reflex, as measured by the frequency of motor spikes, and, conversely, depolarizing it can enhance a reflex. Therefore, any inputs, whether central or peripheral in origin, that alter the membrane potential of these interneurons will also change the gain of the local reflexes. From these obscrvations flow the following predictions, which can now be tested. First, because a local reflex of one segment must be related to the movements performed by other segments, the signals providing this information might be expected to converge on the nonspiking interneurons. Second, the output of the nonspiking interneurons might be used to modulate the afferent connections with motor neurons so that a voluntary movement can proceed in the face of conflicting sensory signals. Third, the nonspiking interneurons might modulate or even gate out reflexes when they are inappropriate.

\section{References}

Bässler, U. (1972a) Der "Kniesehnenreflex" bei Carausius morosus: Ubergangsfunktion und Frequenzgang. Kybernetik 11: 32-50.

Bässler, U. (1972b) Der Regelkreis des Kniesehnenreflexes bei der Stabheuschrecke Carausius morosus: Reaktionen auf passive Bewegungen der Tibia. Kybernetik 12: 8-20.

Bässler, U. (1976) Reversal of a reflex to a single motoneuron in the stick insect Carausius morosus. Biol. Cybern. 24: 47-49.

Bässler, U. (1986) Afferent control of walking movements in the stick insect Cuniculina impigra. II. Reflex reversal and the release of the swing phase in the restrained foreleg. J. Comp. Physiol. 158: 351362.

Brogan, R. T., and R. M. Pitman (1981) Axonal regeneration in an identified insect motoneurone. J. Physiol. (Lond.) 319: 34P-35P.

Burns, M. D. (1974) Structure and physiology of the locust femoral chordotonal organ. J. Insect Physiol. 20: 1319-1339.

Burrows, M. (1979) Synaptic potentials effect the release of transmitter from locust nonspiking interneurons. Science 204: 81-83.

Burrows, M. (1980) The control of sets of motoneurones by local interneurones in the locust. J. Physiol. (Lond.) 298: 213-233.

Burrows, M. (1987a) Parallel processing of proprioceptive signals by spiking local interneurons and motor neurons in the locust. J. Neurosci. 7: 1064-1080.

Burrows, M. (1987b) Inhibitory interactions between spiking and nonspiking local interneurons in the locust. J. Neurosci. 7: 3282-3292.

Burrows, M., and G. A. Horridge (1974) The organization of inputs to motoneurones of the locust metathoracic leg. Phil. Trans. R. Soc. Lond. [Biol.] 269: 49-94.

Burrows, M., and M. V.S. Siegler (1978) Graded synaptic transmission between local interneurones and motoneurones in the metathoracic ganglion of the locust. J. Physiol. (Lond.) 285: 231-255.
Burrows, M., and M. V. S. Siegler (1982) Spiking local interneurons mediate local reflexes. Science 217: 650-652.

Campbell, J. I. (1961) The anatomy of the nervous system of the mesothorax of Locusta migratoria migratorioides. Proc. Zool. Soc. Lond. 137: 403-432.

Cruse, $H$. (1981) Is the position of the femur-tibia joint under feedback control in the walking stick insect? I. Force measurements. J. Exp. Biol. 92: 87-95.

Cruse, H., and H. J. Pflüger (1981) Is the position of the femur-tibia joint under feedback control in the walking stick insect? II. Electrophysiological recordings. J. Exp. Biol. 92: 97-107.

Field, L. H., and M. Burrows (1982) Reflex effects of the femoral chordotonal organ upon leg motor neurones of the locust. J. Exp. Biol. 101: $265-285$.

Field, L. II., and F. C. Rind (1981) A single insect chordotonal organ mediates inter- and intra-segmental leg reflexes. Comp. Biochem. Physiol. 68A: 99-102.

Hofmann, T., U. T. Koch, and U. Bässler (1985) Physiology of the femoral chordotonal organ in the stick insect, Cuniculina impigra. J. Exp. Biol. 114: 207-223.

Laurent, G. J. and M. Burrows (1988) Direct excitation of nonspiking local interneurones by exteroceptors underlies tactile reflexes in the locust. J. Comp. Physiol. 162: 563-572.

Siegler, M. V. S. (1981a) Posture and history of movement determine membrane potential and synaptic events in nonspiking interneurons and motor neurons of the locust. J. Neurophysiol. 46: 296-309.

Siegler, M. V. S. (1981b) Postural changes alter synaptic interactions between nonspiking interneurons and motor neurons of the locust. J. Neurophysiol. 46: 310-323.

Siegler, M. V. S., and M. Burrows (1984) The morphology of two groups of spiking local interneurones in the metathoracic ganglion of the locust. J. Comp. Neurol. 224: 463-482.

Tyrer, N. M., and G. E. Gregory (1982) A guide to the neuroanatomy of locust suboesophageal and thoracic ganglia. Phil. Trans. R. Soc. Lond. [Biol.] 297: 91-123.

Usherwood, P. N. R., H. I. Runion, and J. I. Campbell (1968) Structure and physiology of a chordotonal organ in the locust leg. I. Exp. Biol. 48: 305-323.

Watkins, B. L., M. Burrows, and M. V. S. Siegler (1985) The structure of locust non-spiking interneurones in relation to the anatomy of their segmental ganglion. J. Comp. Neurol. 240: 233-255.

Zill, S. N. (1981) Central modulation of reflex effects of the locust metathoracic femoral chordotonal organ. Soc. Neurosci. Abstr. 7: 252.

Zill, S. N. (1985a) Plasticity and proprioception in insects. I. Responses and cellular properties of individual receptors of the locust metathoracic femoral chordotonal organ. J. Exp. Biol. 116: 435-461.

Zill, S. N. (1985b) Plasticity and proprioception in insects. II. Modes of reflex action of the locust metathoracic femoral chordotonal organ. J. Exp. Biol. 116: 463-480.

Zill, S. N. (1987) Selective mechanical stimulation of an identified proprioceptor in freely moving locusts: Role of resistance reflexes in active posture. Brain Res. 417: 195-198. 